\title{
DIELECTRIC RELAXATION STUDIES ON THE BINARY MIXTURE OF DIETHYLENE GLYCOL AND ACETO NITRILE/ACRYLONITRILE IN NON-POLAR SOLVENT
}

\author{
M. Subramanian ${ }^{1}$, G. Parthipan,", and R. M. Uma Maheshwari ${ }^{3}$ \\ ${ }^{1}$ Dean, Fatima Michael College of Engineering and Technology, \\ Madurai-625020, Tamil Nadu, India \\ ${ }^{2}$ Department of Physics, Vel Tech Multitech DR Rangarajan DR Sakunthala \\ Engineering college, Avadi, Chennai - 600 055. Tamilnadu, India \\ ${ }^{3}$ Department of Physics, Sree Sevugan Annamalai College, Devakottai, Tamilnadu, India \\ *E-mail: phdparthipan@gmail.com
}

\begin{abstract}
The permittivity and the dielectric loss for the ternary mixtures of diethylene glycol and acetonitrile / acrylonitrile in 1,4 dioxan have been obtained at an equimolar concentration at $35^{\circ} \mathrm{c}$. The distribution parameter $(\alpha)$, the most probable relaxation time $(\tau)$, the relaxation time for overall rotation of the molecule $\left(\tau_{1}\right)$, the relaxation time for group rotation $\left(\tau_{2}\right)$ and the excess dipole moment $(\Delta \mu)$ for various systems have been calculated by Higasi's method and Cole-Cole method also utilized. Among the two systems, the size of the molecules is more for the mixture of diethylene glycol and acetonitrile. Negative values of dipolar increment are obtained for both studied systems and interpreted according to the results.

Keywords: dielectric constant, dielectric loss, relaxation time, viscosity, H-Bond, activation free energy, excess dipole moment.
\end{abstract}

(C) RASĀYAN. All rights reserved

\section{INTRODUCTION}

Molecular dynamics for the liquid system can be studied by many methods such as ultrasonic, spectroscopic and dielectric method etc ${ }^{1-3}$. The dielectric technique has three different methods to identify the existing molecular interaction in the liquids mixture to be studied. They are Kirkwood correlation parameter studies, dipolar incremental studies, and relaxation studies. The majority of the researchers have been using relaxation method because of its ability to give the information on size and shape of the molecule. When the high frequency of the electric field is applied to liquids mixture, the molecules in the mixture are disturbed. So molecules move from their original position to new position and again it comes to the original position due to alternate current. Time for reaching the molecule from a new position to original position is called as relaxation time and the mechanism is known as relaxation mechanism. This dielectric relaxation mechanism leads to an anomalous dispersion of dielectric constants that decrease with the increase of frequency. Hence, the study of dielectric relaxation would give information on the fluid structure. Debye narrated that dielectric relaxation as the time lag experienced by a dipolar molecule to orient itself towards to direction of high-frequency field against the viscous forces of its surroundings ${ }^{4}$. Nature of the functional groups and the volume of the molecules decide the value of relaxation time of a liquid to be investigated. Sengwa et al studied the characterization of conductivity relaxation processes for the binary mixtures of propylene carbonate with acetonitrile based on charge dynamics and hydrogenbond molecular interactions 5 . Mehrotra et al carried out the temperature dependent dielectric relaxation for the mixture of acetonitrile and chlorobenzene ${ }^{6}$. Dielectric and dynamic mechanical relaxation studies of styrene-acrylonitrile copolymers was reported by Cook et $a l^{7}$.

Rasayan J. Chem., 11(3), 935-941(2018)

http://dx.doi.org/10.31788/RJC.2018.1131879

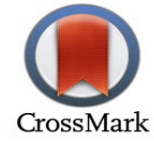


Chaube et al reported the relaxation studies of a binary mixture of diethylene glycol and methnal ${ }^{8}$. The following ternary systems are taken for the investigation at $308 \mathrm{~K}$ :

System 1: diethylene glycol + acetonitrile in 1,4 dioxan

System 2 : diethylene glycol + acrylonitrile in 1,4 dioxan

The literature survey says that the relaxation and viscous studies for above-cited systems have not been carried out so far. Diethylene glycol is a compound which can be used to manufacture of unsaturated polyester resins, polyurethanes, and plasticizers. It is a solvent for resins, oils nitrocellulose, dyes and other organic compounds. DEG is also used as brake fluid, lubricants, wallpaper strippers, artificial fog, haze solutions and personal care products. In the purification of butadiene in refineries and manufacture of pharmaceuticals and photographic film, acetonitrile is used as a solvent. Due to its high dielectric constant and dissolving nature, it is extensively used for battery applications. Acrylonitrile is used mostly as a raw material for the fabrication of acrylic and modacrylic fibers. It also takes part in a significant role in the manufacturing of plastics, adiponitrile, rubbers styrene-acrylonitrile, acrylamide and nitrile barrier resins.

\section{EXPERIMENTAL}

The static dielectric constants and dielectric constant at high frequency have been calculated from the capacitance and refractive index measurements respectively. VLCR-7 meter supplied by Vasavi Electronics, India is used for the capacitance measurement ${ }^{9}$. $\varepsilon_{\infty}$ was taken as the square of the refractive index $\left(\mathrm{n}_{\mathrm{D}}\right)$, which was measured by Abbe's refractometer ${ }^{10}$. With help of $10 \mathrm{ml}$ specific gravity bottle and a K-Roy microbalance, concentration and densities for corresponding concentration were determined. Chemicals used in the investigation are BDH AnalaR variety purified by standard methods. The uncertainties in the measurements of dielectric constants and refractive indices were \pm 0.0005 and \pm 0.0002 respectively. X-band test bench was used to measure the dielectric constant and dielectric loss of liquids. K-27 Klystron, supplied by the Scientific Instrument Co. Ltd., Allahabad was used as the source for microwave power at $8.33 \mathrm{GHz}$. Experiments were done at a constant temperature $\left(35^{\circ} \mathrm{c}\right)$. The temperature was controlled by thermostat ${ }^{11}$.

Resonance circuit methods, transmission line methods, impedance bridge methods and free space techniques are the methods used to measure dielectric constants at microwave frequencies. Hill et al. critically analyzed the merits and demerits of the above methods ${ }^{12}$. In the present study, the transmission line method using wave-guide technique is employed.

To measure the dielectric constant and dielectric loss of the solutions of polar liquids in non-polar solvents, Smyth et al. have described a 'width at twice minimum' standing wave ratio method ${ }^{13}$. For short circuit termination of dielectric lengths of integral multiples of $\left(\lambda_{d} / 2\right)$ the inverse voltage standing wave, the ratio is given by:

$$
\rho_{n}=z_{2} \tanh \left(\frac{n \alpha_{d} \lambda_{d}}{2}\right)
$$

Where, $Z_{2}=\left(\frac{\lambda_{d}}{\lambda_{g}}\right)$ For the low loss of liquids $\alpha$ is small and the Eq.(1) can be modified as:

$$
\begin{aligned}
\rho_{n} & =z_{2} \tanh \left(\frac{n \alpha_{d} \lambda_{d}}{2}\right) \\
& =\frac{\lambda_{d}}{\lambda_{g}}\left(\frac{n \alpha_{d} \lambda_{d}}{2}\right)
\end{aligned}
$$




$$
=\frac{n \alpha_{d} \lambda_{d}^{2}}{2 \lambda_{g}}
$$

The experimentally measured value of $\rho_{n}$ will include a factor $R_{s c}$ to account for the plunger resistance and other losses.

So, $\quad \rho_{\text {measured }}=\frac{n \alpha_{d} \lambda_{d}^{2}}{2 \lambda_{g}}+R_{s c}$

The loss factor $R_{s c}$ due to the plunger resistance may be eliminated by plotting several values of $\rho_{\text {measured }} v S \mathrm{n}$. From the graph, the slope gives the value of $\alpha_{d}$.

$$
\alpha_{\mathrm{d}}=\left(\frac{2 \lambda_{g}}{\lambda_{d}^{2}}\right)\left(\frac{d \rho_{\text {measured }}}{d n}\right)
$$

The dielectric loss $\varepsilon^{\prime}$ will be given by:

$$
\begin{aligned}
\mathcal{E}^{\prime \prime} & =\frac{1}{\pi}\left(\frac{\lambda_{0}^{2}}{\lambda_{d}}\right) \alpha_{d} \\
& =\frac{1}{\pi}\left(\frac{\lambda_{0}^{2}}{\lambda_{d}}\right)\left(\frac{2 \lambda_{g}}{\lambda_{d}^{2}}\right)\left(\frac{d \rho_{\text {measured }}}{d n}\right) \\
& =\frac{2}{\pi}\left(\frac{\lambda_{0}^{2} \lambda_{g}}{\lambda_{d}^{3}}\right)\left(\frac{d \rho_{\text {measured }}}{d n}\right)
\end{aligned}
$$

The dielectric constant $\varepsilon^{\prime}$ is given by:

$$
\varepsilon=\left(\frac{\lambda_{o}}{\lambda_{c}}\right)^{2}+\left(\frac{\lambda_{o}}{\lambda_{d}}\right)^{2}
$$

\section{Cole-Cole Plot}

Cole and Cole proposed a semi-empirical formula in the case of associated liquids ${ }^{14}$.

$$
\varepsilon^{*}=\varepsilon^{\prime}-j \varepsilon^{\prime \prime}=\varepsilon_{\infty}+\frac{\left(\varepsilon_{o}-\varepsilon_{\infty}\right)}{(1-j \omega \tau)^{(1-\alpha)}}
$$

Where, $\alpha$ is an empirical constant with a value between 0 and 1 . This leads to a depressed semi-circle when $\varepsilon^{\prime \prime}$ is plotted against $\varepsilon^{\prime}$. Also, a most frequently encounter experimental plot in the complex plane locus for associated liquids leads to a right-skewed arc (Fig-1), which may be represented by a function.

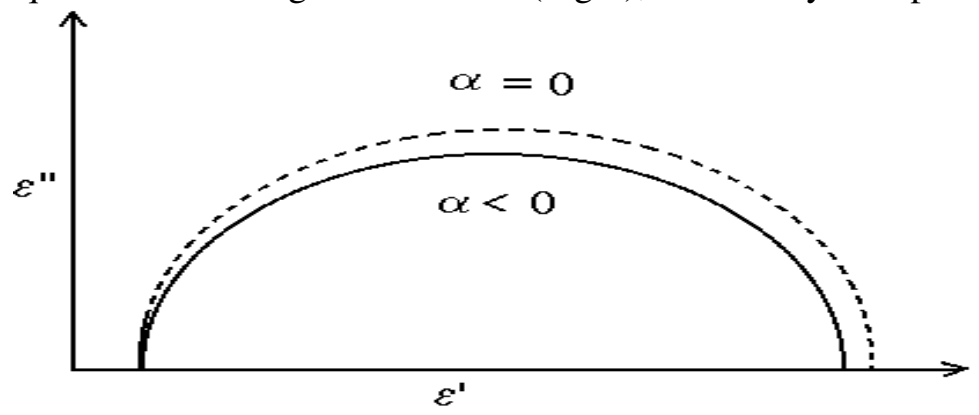

Fig.-1: The Cole-Cole plot 


$$
\varepsilon^{*}=\varepsilon^{\prime}-j \varepsilon^{\prime \prime}=\varepsilon_{\infty}+\frac{\left(\varepsilon_{o}-\varepsilon_{\infty}\right)}{(1+j \omega \tau)^{\beta}}
$$

Where, $\beta$ is also an empirical constant with a value between 0 and 1 .

When $\alpha=0$ in Eq. ( 6 ) or $\beta=1$ in Eq. (7), the Eq. becomes identical with

$$
\varepsilon^{\prime \prime}=\varepsilon_{\propto}+\frac{\left(\varepsilon_{o}-\varepsilon_{\infty}\right)}{(1+j \omega \tau)}
$$

A large variety of functions for representation of the distribution of relaxation time were proposed on an empirical basis, when two or more relaxation processes occur, the absorption regions for the two processes overlap and an appreciable value of $\alpha$ occurs $^{15}$.

The measured values of $\varepsilon^{\prime}, \varepsilon^{\prime \prime}, \varepsilon_{\mathrm{o}}$ and $\varepsilon_{\infty}$ were used in Cole-Cole Plot. From the plot, the relaxation time $\tau$ can be obtained by using the equation given below ${ }^{16}$ :

$$
(\omega \tau)^{1-\alpha}=\left(\frac{v}{u}\right)
$$

Where, $\omega$ be the angular frequency $\alpha$ be the distribution parameter and on the Cole-Cole plot, $v$ is the distance between $\varepsilon_{0}$ and experimental point and $u$ is the distance between $\varepsilon_{\infty}$ and that point.

\section{Higasi's Method}

Smyth and co-workers gives the best fit for the study of solutions ${ }^{17}$. In their studies the values of $\mathcal{\varepsilon}, \varepsilon^{\prime \prime}$, $\varepsilon_{o}$ and $\varepsilon_{\infty}$ are understood to vary linearly with weight fraction $w_{2}$ of the solute, one can write:

$$
\left.\begin{array}{ll}
\mathcal{E}_{0}=\varepsilon_{1}+a_{0} w_{2} & \\
\mathcal{E}^{\prime}=\varepsilon_{1}+a^{\prime} w_{2} & \\
\mathcal{E}^{\prime \prime}=a^{\prime \prime} w_{2} & \\
\mathcal{E}_{\infty}=\varepsilon_{1 \infty}+a_{\infty} w_{2} & \}
\end{array}\right\}
$$

Where $a_{0}, a^{\prime}, a^{\prime}$ and $a_{\infty}$ are constants. Higashi derived a relation connecting $\tau$ and $\alpha$.

$$
\begin{aligned}
& \tau=\frac{1}{\omega}\left(\frac{\mathrm{A}^{2}+\mathrm{B}^{2}}{C^{2}}\right)^{\frac{1}{2(1-\alpha)}} \\
& (1-\alpha)=\frac{2}{\pi} \tan ^{-1}\left(\frac{A}{B}\right)
\end{aligned}
$$

Where

$$
\begin{aligned}
& A=a^{\prime \prime}\left(a_{0}-a_{\infty}\right) \\
& B=\left(a_{0}-a^{\prime}\right)\left(a^{\prime}-a_{\infty}\right)-a^{\prime 2} \\
& C=\left(a^{\prime}-a_{\infty}\right)^{2}+a^{\prime 2}
\end{aligned}
$$

The Debye equation in terms of $\mathrm{a}_{0}, \mathrm{a}, \mathrm{a}$ " and $\mathrm{a}_{\infty}$ give in two independent equations ${ }^{18}$ :

$$
\begin{array}{r}
\tau_{1}=\frac{a^{\prime \prime}}{\omega\left(a^{\prime}-a_{\infty}\right)} \\
\tau_{2}=\frac{a_{o}-a^{\prime}}{\omega a^{\prime \prime}}
\end{array}
$$

The mean relaxation time $\tau$ is given as:

$$
\tau=\sqrt{\tau_{1} \tau_{2}}
$$


RASĀYAN J. Chem.

Vol. 11 | No. 3 |935 - 941 | July - September | 2018

The dipole moment is calculated by Higasi's relation:

$$
\mu=\left(\frac{27 M_{2} k T}{\pi N_{A} d_{1}}\right)^{1 / 2}\left(\frac{1}{\left(2 \varepsilon_{1}+2\right)}\right)
$$

$\tau_{1}$ given by the eq. (14) may be described as the relaxation time for overall rotation of the molecules and $\tau_{2}$ given by the equation (15) may be described as relaxation time for functional group rotations. In this paper, the relaxation mechanisms involved in the solutions of alcohol and nitriles have been studied by both Cole-Cole and Higasi's methods.

\section{RESULTS AND DISCUSSION}

The values of the different frequency based dielectric constants, the dielectric loss, the distribution parameter $(\alpha)$, the relaxation time for overall rotation of the molecule $\left(\tau_{1}\right)$, the relaxation time for group rotation $\left(\tau_{2}\right)$, the most probable relaxation time $(\tau)$, and the excess dipole moment for various systems are given in Tables-1 and 2. Higashi's parameters and Cole -Cole's parameters were calculated using appropriate equations.

Dielectric constant at static and high frequencies increases with increasing the concentration of nitrile in both systems and similarly dielectric constant at microwave frequency, dielectric loss and all related parameters are varying with concentrations which can be seen in Table-1 and 2. It is concluded that there is an interaction between either nitrile with nitrile or nitrile with diethylene glycol ${ }^{16}$.

Table-1: Values of Dielectric Constants and Higasi's Parameters at 308K

\begin{tabular}{c|c|c|c|c|c|c|c|c|c}
\hline System & $\mathrm{X}_{2}$ & $\varepsilon_{o}$ & $\varepsilon_{\infty}$ & $\varepsilon^{\prime}$ & $\mathcal{E}^{\prime \prime}$ & $a_{0}$ & $a_{\infty}$ & $a^{\prime}$ & $a^{\prime}$ \\
\hline diethylene & 0.05 & 2.8590 & 2.0150 & 2.7405 & 0.1972 & 13.3404 & 0.2176 & 10.9700 & 3.9433 \\
glycol + & 0.06 & 2.8823 & 2.0141 & 2.7538 & 0.2049 & 11.5048 & 0.1671 & 9.3626 & 3.4154 \\
acetonitrile & 0.07 & 2.9056 & 2.0133 & 2.7649 & 0.2125 & 10.1937 & 0.1311 & 8.1844 & 3.0356 \\
+1,4dioxan & & & & & & & & & \\
\hline diethylene & 0.05 & 2.8590 & 2.0127 & 2.7427 & 0.1915 & 13.3404 & 0.1722 & 11.0140 & 3.8294 \\
glycol + & 0.06 & 2.8823 & 2.0136 & 2.7560 & 0.1992 & 11.5048 & 0.1577 & 9.3997 & 3.3197 \\
acrylonitrile+ & 0.07 & 2.9056 & 2.0144 & 2.7694 & 0.2070 & 10.1937 & 0.1473 & 8.2485 & 2.9575 \\
1,4dioxan & & & & & & & & & \\
\hline
\end{tabular}

Davidson stated that any system will have types of relaxation time such as intermolecular relaxation time $\left(\tau_{1}\right)$ and intramolecular relaxation time $\left(\tau_{2}\right)$ when the ratio of the two relaxation times $\left(\tau_{1} / \tau_{2}\right)$ is greater than $\operatorname{six}^{19}$. In this investigation, no such resolution is originated to occur due to the enhanced overlap of two nearly equal regions. The different sizes of the molecules have different relaxation times. The increase of $\tau_{1}$ and $\tau_{2}$ with dilution is assigned to the enhancement in the size of the molecules due to both inter and intramolecular interactions. The coupling between the dipoles is also increased by interaction which hindrance rotating of the dipoles in the mixture ${ }^{16} . \tau$ ' values obtained by the Cole-Cole method are slower than the values obtained by the Higashi method. This discrepancy is due to different methods ${ }^{18}$. Relaxation time for both studied system varies with concentration indicates the existence of an interaction between the molecules which can be seen in the Table-2. The values of $\tau_{1}$ are higher in a mixture of diethylene glycol + acetonitrile in 1,4 dioxan than in the mixture of diethylene glycol + acrylonitrile in 1,4 dioxan .

This is due to the increased size of the molecules in the mixture of diethylene glycol and acetonitrile than the mixture of diethylene glycol and acrylonitrile. Added, it can be said that acetonitrile interacts alcohol more than acrylonitrile.

But in the case of $\tau_{2}$, the value of $\tau_{2}$ is higher for the mixture of diethylene glycol and acrylonitrile than a mixture of diethylene glycol and acetonitrile. This difference in $\tau_{2}$ is due to the chain length of the 
acrylonitrile which is a little bit bigger than acetonitrile. Similar results are reported by Purohit et $a l^{20}$. for the mixture of diethylene glycol with monomethyl, monoethyl and monobutyl ether.

The viscosity of both studied system is more or less equal because both are aliphatic nitriles. Even though both systems are having nearly equal viscosity nature, $\tau$ values are different. This signifies that the value of $\tau$ is independent to the viscosity of the mixtures. Due to the large size of the solute molecules, the value of $\tau$ increases ${ }^{20}$.

Table-2:Values of Relaxation Time, a Distribution Parameter, and Activation Energy at 308K

\begin{tabular}{|c|c|c|c|c|c|c|c|c|c|c|}
\hline \multirow{3}{*}{ System } & \multirow{3}{*}{$\mathrm{X}_{2}$} & \multicolumn{4}{|c|}{ Relaxation time ( pico second) } & \multicolumn{2}{|c|}{$\begin{array}{l}\text { Distribution } \\
\text { parameter }\end{array}$} & \multirow{2}{*}{\multicolumn{2}{|c|}{$\begin{array}{c}\text { Activation } \\
\text { energy } \\
\text { ( KJ / mole ) }\end{array}$}} & \multirow{3}{*}{$\begin{array}{c}\begin{array}{c}\text { Change } \\
\text { In } \\
\text { Dipole } \\
\text { moment }\end{array} \\
\Delta \mu\end{array}$} \\
\hline & & \multicolumn{3}{|c|}{ Higashi method } & \multirow{2}{*}{$\begin{array}{c}\begin{array}{c}\text { Cole- } \\
\text { Cole } \\
\text { plot }\end{array} \\
\tau\end{array}$} & \multirow{2}{*}{$\begin{array}{c}\text { Higashi } \\
\frac{\alpha}{}\end{array}$} & \multirow{2}{*}{$\begin{array}{c}\begin{array}{c}\text { Cole- } \\
\text { Cole }\end{array} \\
\alpha\end{array}$} & & & \\
\hline & & $\tau_{1}$ & $\tau_{2}$ & $\tau$ & & & & $\Delta F_{\tau}$ & $\Delta F_{\eta}$ & \\
\hline diethylene & 0.05 & 6.06 & 9.94 & 5.86 & 3.92 & 0.12 & 0.19 & 9.10 & 13.36 & -4.25 \\
\hline glycol + & 0.06 & 6.14 & 10.37 & 5.95 & 4.07 & 0.13 & 0.20 & 9.14 & 13.39 & -4.36 \\
\hline $\begin{array}{l}\text { acetonitrile } \\
+1,4 \text { dioxan }\end{array}$ & 0.07 & 6.23 & 10.94 & 6.06 & 4.23 & 0.14 & 0.21 & 9.18 & 13.41 & -4.44 \\
\hline diethylene & 0.05 & 5.84 & 10.05 & 5.59 & 3.64 & 0.13 & 0.21 & 8.98 & 13.38 & -4.32 \\
\hline glycol + & 0.06 & 5.94 & 10.49 & 5.70 & 3.70 & 0.14 & 0.21 & 9.03 & 13.41 & -4.44 \\
\hline $\begin{array}{l}\text { acrylonitrile } \\
+1,4 \text { dioxan }\end{array}$ & 0.07 & 6.04 & 10.88 & 5.82 & 3.85 & 0.15 & 0.22 & 9.08 & 13.41 & -4.53 \\
\hline
\end{tabular}

$\Delta F_{\tau}$ increases with increasing the concentration of nitrile in both system because of the chain length of the aliphatic nitriles and the intermolecular interaction between nitrile and alcohol. This gives rise to a big size molecules ${ }^{21}$. The larger size of the molecules needs much energy to lift a molecule above the potential energy barrier. Increase in the value of $\Delta F_{\tau}$ decreases the probability of a jump from one orientation into another resulting in the increase in the value of the relaxation time ${ }^{21}$. In all both systems, the free energy of activation of dipole orientation $\Delta F_{\tau}$ is less than the corresponding value of the viscous force $\Delta F_{\eta}$. It is expected because the dielectric relaxation process involves only rotational motion while viscous flow involves translation as well as rotational motion ${ }^{21}$.

Presence of hydrogen bond in the ternary systems can be identified by excess dipole moment which can be calculated by using Eq.(9). Using Debecker and Huyskens equation, the excess dipole moment is calculated $^{21}$, as:

$$
\Delta \mu=\mu_{a b}-\mu_{a}-\mu_{b}
$$

The presence of a hydrogen bond between the nitrogen of the nitriles with the oxygen of the hydroxy groups of alcohols ie both systems is indicated by the excess dipole moment values as reported by Shobanadri et $a l^{21,22}$. The excess dipole moment may be attributed to the proton-transfer in this bond. Similar results were informed by Thenappan for the mixture of amines and alcohols in benzene and Subramanian for the mixture of alcohols and nitriles in benzene $/ 1,4$ dioxan ${ }^{21,23}$.

Both systems have a negative value of excess dipole moment which implies the absence of ionic structures. The negative value of $\Delta \mu$ also points out the presence of hydrogen bonds between the two different molecules in the mixture ${ }^{24,25}$.

\section{CONCLUSION}

Dielectric relaxation and viscous behaviors of the ternary mixtures of equimolar concentrations of diethylene glycol with acetonitrile/acrylonitrile in 1, 4-dioxan has been studied. This investigation shows that $\tau$ values for diethylene glycol and acetonitrile are slightly greater than the mixtures of diethylene glycol and acrylonitrile. The free energy of activation of dipole orientation $\Delta F_{\tau}$ is less than the 
RASĀYAN $J$. Chem.

Vol. 11 | No. 3 | 935 - 941 | July - September | 2018

corresponding value of the viscous force $\Delta F_{\eta}$ for both systems. Negative values of dipolar increment are obtained for all studied systems, which indicate the absence of ionic structures.

\section{REFERENCES}

1. G. P. Dubey, M. Sharma, and S. Oswal, Journal of Chemical Thermodynamics,41, 849 (2009), DOI: 10.1016/j.jct.2009.02.002.

2. P. Undre, S. N. Helambe, S. B. Jagdale, P. W. Khirade and S. C. Mehrotra, Journal of Molecular Liquids, 137,147(2008), DOI: 10.1016/j.molliq.2007.06.004.

3. J. Barthel, R. Buchner, P.N. Eberspächer, M. Münsterer, J. Stauber and B. Wurm, Journal of Molecular Liquids,78, 83 (1998), DOI: 10.1016/S0167-7322(98)00085-

4. Debye P, 1929, Polar Molecules, Dover, New York .

5. S. Choudhary, P. Dhatarwal and R. J. Sengwa, Journal of Molecular Liquids, 231,491(2007), DOI:10.1016/j.molliq.2017.02.036.

6. I. G. Shere, V. P. Pawar and S. C. Mehrotra, Journal of Molecular Liquids, 133,116(2007), DOI:10.1016/j.molliq.2006.07.002.

7. M. Cook and G. Williams, Polymer, 16, 835(1975), DOI: 10.1016/0032-3861(75)90117-2.

8. H. A. Chaube, V. A. Rana, P. Hudge and .C. Kumbharkhane, Journal of Molecular Liquids, 211,346(2015), DOI: 10.1016/j.molliq.2015.07.020

9. G. Parthipan, H. Aswathaman, U. Sankar and T. Thenappan, Journal of Molecular Liquids, 140, 1 (2008), DOI:10.1016/j.molliq.2007.12.004.

10. G. Parthipan and T. Thenappan, Philosophical Magazine Letters, 89, 82(2009), DOI: 10.1080/09500830902776943

11. G. Parthipan, H. Aswathaman, G. Arivazhagan and T. Thenappan, Philosophical Magazine Letters ,88, 251(2008), DOI: 10.1080/09500830801901832

12. N. E. Hill, W.E. Vaughen, A. H. Price and M. Davies, 1969, Dielectric Properties and Molecular Behavior, Van Nostrand, New York

13. A. D. Franklin, W.M. Heston, E. J. Jr Hennelly and C.P Smyth, Journal of American Chemical Society, 72, 3443(1950)

14. K. S. Cole and R. H. Cole, Journal of Chemical Physics, 9, 341(1941)

15. H. Frohlich, 1958, Theory of Dielectrics, Clarendon Press, Oxford .

16. G. Parthipan and P. Anandan, Journal Non-Crystalline Solids, 356, 1721(2010), DOI: 10.1016/j.jnoncrysol.2010.06.023

17. K. Higasi, Y. Koya and M. Nakamura, Bulletin of Chemical Society Japan., 44, 988(1971)

18. G. Parthipan, G. Arivazhagan, M. Subramanian and T. Thenappan, Physics and Chemistry of Liquids, 49, 1(2011), DOI: 10.1080/00319100902894231

19. D. W. Davidson and R. H. Cole. Journal of Chemical Physics ,19, 1484(1951)

20. H. D. Purohit and R.J Sengwa, Journal of Molecular Liquids, 40, 237(1989), DOI: 10.1016/01677322(89)80057-1

21. M. Subramanian, T. Thenappan and G. Parthipan. PhilosophicalMagazineLetters , 88, 889 (2008), DOI: $10.1080 / 09500830802498986$

22. V. Madhurima, N. Satyan, V.R.K. Murthy and J. Sobhanadri, Indian Journal of Pure and Applied Physics, 36, 144(1998)

23. T. Thenappan and M. Subramanian, Material Scence and Engineering B, 86, 7(2001), DOI: 10.1016/S0921-5107(01)00565-7

24. M. Subramanian and G. Parthipan, Journal of Adv Research in Dynamical \& Control Systems, 10,599(2018)

25. M. Subramanian and G. Parthipan, Research Journal of Pharmacy and Technology, 11,761(2018), DOI: 10.5958/0974-360X.2018.00143.9

[RJC-1879/2017] 